\title{
Possible association between haplotypes of the FKBP5 gene and suicidal bipolar disorder, but not with melancholic depression and psychotic features, in the course of bipolar disorder
}

\author{
This article was published in the following Dove Press journal: \\ Neuropsychiatric Disease and Treatment \\ 3 February 2014 \\ Number of times this article has been viewed
}

\author{
Anna Leszczyńska- \\ Rodziewicz' \\ Aleksandra \\ Szczepankiewicz ${ }^{2}$ \\ Beata Narożna ${ }^{2}$ \\ Maria Skibińska' \\ Joanna Pawlak' \\ Monika Dmitrzak-Węglarz \\ Joanna Hauser' \\ 'Psychiatric Genetics Unit, \\ ${ }^{2}$ Laboratory of Molecular and Cell \\ Biology, Department of Pulmonology, \\ Pediatric Allergy and Clinical \\ Immunology, Poznań University of \\ Medical Sciences, Poznań, Poland
}

\begin{abstract}
We aimed to analyzed the association between polymorphisms of the FKBP5 (FK506 binding protein 5) gene and subtypes of bipolar disorder. In the study, we included 195 bipolar disorder patients with psychotic features, 129 bipolar patients with melancholic depression, and 156 bipolar patients with a history of suicidal attempts. We found weak association between the haplotypes of the FKBP5 gene and bipolar patients with suicidal attempts. We did not find an association between polymorphisms of the FKBP5 gene and bipolar disorder with psychotic features, nor with bipolar disorder with melancholic depression. Limitations of our study are the absence of data about previous trauma exposure and the small sample size of patients, which of course can lead to false-positive results, so further validation and replication of the present findings are still needed.
\end{abstract}

Keywords: FKBP5 gene, bipolar disorder, melancholic depression, psychotic features, suicide attempts

\section{Introduction}

Bipolar disorder is thought to originate from the interactions between susceptibility genes and adverse environmental stresses. The hypothalamus-pituitary-adrenal (HPA) axis is a major system involved in stress response, and its dysregulation is an important element in the pathogenesis of affective disorders. ${ }^{1}$ A dysregulation of the stress hormone is one of the most potent biological findings in stress-related disorders, including depression, especially melancholic depression. Stress is also seen as one of the factors predisposing both to occurrence of psychotic features and suicidal attempts.

HPA-axis reactivity is regulated by negative-feedback mechanisms induced by mineralocorticoid and glucocorticoid receptors. The activity of the glucocorticoid receptor (GR) is regulated by the FKBP5 (FK506 binding protein 5) gene. ${ }^{2}$ The binding of FKBP5 to the GR complex decreases the affinity of cortisol binding, followed by a deficient receptor nuclear translocation, and therefore reduces GR sensitivity. ${ }^{3}$ GR activation elicits expression of FKBP5 messenger ribonucleic acid and protein and this yield to ultrashort feedback loop, affecting GR sensitivity. ${ }^{4}$ Alterations in the HPA axis in response to stress may be associated with functional polymorphisms in genes encoding those receptors. ${ }^{5,6}$ Menke et al ${ }^{4}$ suggested that depressed patients carrying the FKBP5 rs1360780 allele T exhibit significant GR resistance compared with healthy controls. The results obtained by Menke et $\mathrm{al}^{4}$ were measured by a
Correspondence: Anna LeszczyńskaRodziewicz

Psychiatric Genetics Unit, Poznań

University of Medical Sciences, 27/33

Szpitalna Street, Poznań 60-572, Poland

Tel +48 6l 849 I3। I

$\mathrm{Fax}+4861848$ 01 II

Email alrodziewicz@ump.edu.pl 
dexamethasone-suppression test, which caused (only in depressed patients) suppression of adrenocorticotropic hormone and plasma cortisol concentrations. ${ }^{4}$

Zimmermann et al showed that there is association between the genotypes of the FKBP5 gene, trauma, and onset of depression. ${ }^{7}$ Other single-nucleotide polymorphisms (SNPs) of the FKBP5 gene - rs4713916, rs1360780, and rs3800373 - are associated with the deficient normalization of stress-induced cortisol secretion in healthy humans after psychosocial stress. ${ }^{8,9}$

In regard to suicide, $F K B P 5$ gene expression was reduced in the amygdala of suicide victims, ${ }^{3}$ and analysis of haplotypes of the FKBP5 gene in suicidal patients showed an association in previous studies..$^{10,11}$

We hypothesized that genetic variation in the FKBP5 gene might be one of the factors influencing dysfunction of the HPA axis and altering the risk as well as the course of bipolar disorder and it subtypes. As there have been few studies performed so far on depressive disorder and suicidal patients, and none to our knowledge on bipolar patients having psychotic features nor with melancholic depression in the course of bipolar disorder, we aimed to analyze the eight polymorphisms in relation to the subtypes of bipolar disorder.

\section{Methods}

\section{Patients}

The study was performed on a group of 528 bipolar patients, among which 195 had a history of psychotic features, 129 had a history of melancholic depression, and 156 had a history of suicide attempts. Patients were recruited from inpatients of the Department of Adult Psychiatry, Poznań University of Medical Sciences, living in the Wielkopolska region of Poland. This region is considered ethnically homogeneous. ${ }^{12}$ The mean age of onset was 31.6 years, standard deviation (SD) 11.5, overall mean number of episodes was eleven, and among those depressive episodes six and manic episodes five. The mean age of patients was 44.36 years, SD 13.9. Consensus lifetime diagnosis by at least two experienced psychiatrists was made for each patient. The phenotyping of patients was based on medical records, family history, and clinical interviews according to Diagnostic and Statistical Manual of Mental Disorders (DSM-IV) criteria using the Structured Clinical Interview for DSM Disorders. ${ }^{13}$

\section{Control group}

The control group consisted of 742 subjects. Control subjects were recruited from the group of healthy volunteers, blood donors, and hospital staff and students of the University of Medical Sciences in Poznań and the Clinical
Neuropsychology Unit, Collegium Medicum Bydgoszcz. Only $40 \%$ of the control group were psychiatrically screened, which is the main limitation of this paper. The mean age was 37.7 years, SD 12.5. After complete description of the study, written informed consent was obtained. The study was performed in compliance with the Declaration of Helsinki and was approved by the local bioethics committee.

\section{Genotyping}

Deoxyribonucleic acid (DNA) was extracted using the salting-out method. ${ }^{14}$ The list of SNPs analyzed and the ID numbers of TaqMan assays are shown in Table 1. SNP selection included the following criteria: high frequency (minor allele frequency $[\mathrm{MAF}]>0.05$; the MAF for our group [shown in Table 2] was the same as in the Caucasian population [CEU population from HapMap] shown in Pub Med), indication as tag SNP in HapMap, or previously reported associations for psychiatric disorders (both positive and negative findings). SNPs chosen covered noncoding regions (introns, untranslated regions) possible to affect gene regulation. The eight polymorphisms were genotyped with TaqMan Genotyping Master Mix and TaqMan SNP genotyping assays (Life Technologies, Carlsbad, CA, USA).

All the assays were validated and predesigned. Reaction components and amplification parameters were based on manufacturer's instructions. The ABI Prism ${ }^{\circledR} 7900$ HT (Life Technologies) sequence-detection system was used for amplification for TaqMan SNP genotyping assay plates. SDS version 2.1 (Life Technologies) software was used for data acquisition and analysis. The same software was used for the allelic discrimination-analysis module.

Plate genomic control DNA samples and nontemplate controls (water) were included for each reaction. The TaqMan SNP genotyping assay was controlled (25\% of randomly chosen samples from both groups) to check for genotyping accuracy. Identical genotypes were identified in all repeated samples. The diagnosis of the subjects during genotyping was not known.

Table I FKBP5 gene polymorphism description

\begin{tabular}{lllll}
\hline SNP & Substitution & Location & Method & Assay ID \\
\hline rs1360780 & $\mathrm{C} / \mathrm{T}$ & Intron 9 & TaqMan & C_8852038_10 \\
rs755658 & $\mathrm{C} / \mathrm{T}$ & Intron 3 & TaqMan & C_594063_20 \\
rs9470080 & $\mathrm{C} / \mathrm{T}$ & Intron I0 & TaqMan & C_92160_10 \\
rs47I3916 & $\mathrm{A} / \mathrm{G}$ & Intron I0 & TaqMan & C_1979246_10 \\
rs7748266 & $\mathrm{C} / \mathrm{T}$ & Intron 8 & TaqMan & C_29802748_10 \\
rs9296158 & $\mathrm{A} / \mathrm{G}$ & Intron 6 & TaqMan & C_1256775_10 \\
rs9394309 & $\mathrm{A} / \mathrm{G}$ & Intron I0 & TaqMan & C_1256794_20 \\
rs3800373 & $\mathrm{A} / \mathrm{C}$ & 3' UTR & TaqMan & C_27489960_10 \\
\hline
\end{tabular}

Abbreviations: SNP, single-nucleotide polymorphism; FKBP5, FK506 binding protein 5; UTR, untranslated region. 


\section{Statistical analysis}

To test for differences in genotypic and allelic distribution, the two-tailed Pearson $\chi^{2}$ test and Fisher's exact test were used. Chi-square was used for $3 \times 2$ contingency tables to compare genotypes (three different) between cases and controls. Fisher's exact test was used for $2 \times 2$ contingency tables to compare allele frequencies between cases and controls. Two-tailed power analysis was also performed, using Statistica version 8.0 (StatSoft, Tulsa, OK, USA). A demo of the InStat 3 (GraphPad Software, La Jolla, CA, USA) program was used to calculate odds ratios. Linkage disequilibrium between polymorphisms of the FKBP 5 gene was examined by pair-wise comparisons of r2 and D' using Haploview version 4.1 (Broad Institute, Cambridge, MA, USA). ${ }^{15}$ The same software was used to haplotype construction using an expectation-maximization algorithm. Correction for multiple testing was performed for multiple comparisons: in haplotype analysis using 10,000 permutations implemented in the Haploview software. Quanto version 1.2.3 with odds-ratio values between 1.1 and 1.4 for two-sided associations was used for power calculations.

\section{Results}

Hardy-Weinberg equilibrium analysis

All the analyzed SNPs were in Hardy-Weinberg equilibrium concordance for the controls. The only SNP of the FKBP5 gene that was not in concordance with the Hardy-Weinberg law (results shown in Table 2) was rs7748266 in the group of patients with psychotic features $(P=0.03)$, as SNPs in HardyWeinberg disequilibrium are less powerful and do not tend to increase false-positive results. ${ }^{16} \mathrm{We}$ did not exclude this SNP from the study.

\section{Genotyping and allele frequencies}

In Table 2, check markers (showing if this marker, eg, SNP, is valid for such analysis as success rate) for subtypes of BP for the FKBP5 gene are shown. The success rates for genotyping were between $96.03 \% 98.97 \%$, and for all the polymorphisms, genotyping error rates were $<1 \%$.

\section{Genetic association analysis}

We found association with haplotypes CCATTGT $(P=0.0123)$ and CCACTAT $(P=0.0235)$ in bipolar patients with suicidal attempts, but not in bipolar patients with

Table 2 Check markers for subtypes of BP for FKBP5 gene

\begin{tabular}{|c|c|c|c|c|c|c|c|c|}
\hline Number & SNP & Pos chromos & ObsHET & PredHET & HWE & $\%$ zgenotyp & MAF & Alleles \\
\hline \multicolumn{9}{|c|}{ Check markers melancholic } \\
\hline I & rs3800373 & 35650454 & 0.366 & 0.366 & 1.0 & 99.2 & $0.24 I$ & $A: C$ \\
\hline 2 & rs755658 & 35657648 & 0.168 & 0.168 & 1.0 & 95.4 & 0.092 & C:T \\
\hline 3 & rs9296158 & 35675060 & 0.365 & 0.373 & 0.5832 & 98.3 & 0.248 & $\mathrm{G}: \mathrm{A}$ \\
\hline 4 & rs7748266 & 35700722 & 0.213 & 0.226 & 0.1241 & 97.3 & 0.13 & $C: T$ \\
\hline 5 & rsl360780 & $357 \mid 5549$ & 0.365 & 0.374 & 0.4929 & 97.4 & 0.249 & C:T \\
\hline 6 & rs9394309 & 35729759 & 0.376 & 0.387 & 0.4897 & 96.4 & 0.262 & $A: G$ \\
\hline 7 & rs9470080 & $357544 \mid 3$ & 0.399 & 0.4 & 0.9398 & 98.9 & 0.277 & $C: T$ \\
\hline 8 & rs4713916 & 35777961 & 0.37 & 0.379 & $0.537 \mid$ & 98.2 & 0.254 & $\mathrm{G}: \mathrm{A}$ \\
\hline \multicolumn{9}{|c|}{ Check markers psychotic } \\
\hline I & rs3800373 & 35650454 & 0.367 & 0.37 & 0.879 & 98.9 & 0.245 & $A: C$ \\
\hline 2 & rs755658 & 35657648 & 0.168 & 0.167 & 1.0 & 95.4 & 0.092 & C:T \\
\hline 3 & rs9296158 & 35675060 & 0.364 & 0.377 & 0.3189 & 98.3 & 0.252 & $\mathrm{G}: \mathrm{A}$ \\
\hline 4 & rs7748266 & 35700722 & 0.222 & 0.24 & 0.0375 & 97.1 & 0.139 & C:T \\
\hline 5 & rsl360780 & 35715549 & 0.369 & 0.38 & 0.4012 & 97.2 & 0.255 & C:T \\
\hline 6 & rs9394309 & 35729759 & 0.391 & 0.396 & 0.7577 & 96.2 & 0.272 & $A: G$ \\
\hline 7 & rs9470080 & $357544 \mid 3$ & 0.408 & 0.408 & 1.0 & 98.7 & 0.285 & $C: T$ \\
\hline 8 & rs4713916 & 35777961 & 0.393 & 0.387 & 0.7322 & 98.1 & 0.262 & $\mathrm{G}: \mathrm{A}$ \\
\hline \multicolumn{9}{|c|}{ Check markers suicide } \\
\hline I & rs3800373 & 35650454 & 0.365 & 0.36 & 0.7891 & 98.9 & 0.235 & $A: C$ \\
\hline 2 & rs755658 & 35657648 & 0.166 & 0.164 & 0.9119 & 95.6 & 0.09 & C:T \\
\hline 3 & rs9296158 & 35675060 & 0.363 & 0.369 & 0.635 & 98.4 & 0.244 & $\mathrm{G}: \mathrm{A}$ \\
\hline 4 & rs7748266 & 35700722 & 0.208 & 0.223 & 0.0643 & 97.2 & 0.128 & $C: T$ \\
\hline 5 & rsl360780 & 35715549 & 0.365 & 0.373 & 0.5708 & 97.3 & 0.248 & C:T \\
\hline 6 & rs9394309 & 35729759 & 0.377 & 0.385 & 0.5996 & 96.8 & 0.26 & $A: G$ \\
\hline 7 & rs 9470080 & $357544 \mid 3$ & 0.401 & 0.399 & 0.9346 & 99.0 & 0.275 & $\mathrm{C}: \mathrm{T}$ \\
\hline 8 & rs4713916 & $3577796 \mid$ & 0.372 & 0.376 & 0.8302 & 98.3 & 0.251 & $\mathrm{G}: \mathrm{A}$ \\
\hline
\end{tabular}

Abbreviations: FKBP5, FK506 binding protein 5; SNP, single-nucleotide polymorphism; BP, bipolar disorder; ObsHET, observed heterozygous; PredHET, predicted heterozygous; MAF, minor allele frequency; HWE, Hardy-Weinberg equilibrium; Pos chromos, chromosomal position; zgenotyp, \% genotyped (=success rate or call rate). 
melancholic depression and not with bipolar patients with psychotic features (shown in Table 3 and Figures 1-3). We did not find any associations with genotypes (data not shown) nor with alleles (shown in Table 4) for the studied polymorphisms.

\section{Discussion}

In our study, we found association between haplotypes of the FKBP5 gene in bipolar patients with suicidal behavior. Since the power for the studied SNPs for our population of bipolar patients was rather low (20\%), we assumed that it would be even lower for the subtypes, and due to the small sample we would consider this association as pretty weak. Notwithstanding, our results are in line with previously published associations between haplotypes of the FKBP5 gene and suicide victims without a history of mental illness at the moment of death. ${ }^{3,11}$ There have also been studies showing associations between polymorphisms of the FKBP5 gene and suicidal behavior in depressed antidepressant-nonresponding adolescents ${ }^{17}$ and childhood trauma victims. ${ }^{10}$ Zimmerman et al reported that interaction between the FKBP5 genotype and trauma is involved in the onset of depression. ${ }^{7}$ Association between the rs 1360780 and rs3800373 polymorphisms of the FKBP5 gene and suicide attempts in Japanese and European populations have been reported. ${ }^{11,17}$

Table 3 Haplotypes of FKBP5 gene and subtypes of BP disorder

\begin{tabular}{lllll}
\hline Haplotype & Freq & $\begin{array}{l}\text { Case: control } \\
\text { frequencies }\end{array}$ & Chi-square & P-value \\
\hline \multicolumn{5}{c}{ Association-haplotypes melancholic } \\
ACGCCAC & 0.718 & $0.719,0.718$ & 0.001 & 0.9809 \\
CCATTGT & 0.117 & $0.094,0.121$ & 1.484 & 0.2232 \\
CTACTGT & 0.087 & $0.100,0.085$ & 0.624 & 0.4294 \\
ACGCCGT & 0.029 & $0.031,0.029$ & 0.038 & 0.8449 \\
CCACTAT & 0.016 & $0.027,0.014$ & 2.263 & 0.1325 \\
CCACTGT & 0.012 & $0.020,0.011$ & 1.466 & 0.2261 \\
Association-haplotypes psychotic & & \\
ACGCCAC & 0.710 & $0.679,0.718$ & 2.193 & 0.1387 \\
CCATTGT & 0.122 & $0.130,0.121$ & 0.24 & 0.6239 \\
CTACTGT & 0.087 & $0.095,0.085$ & 0.383 & 0.5362 \\
ACGCCGT & 0.031 & $0.038,0.029$ & 0.926 & 0.336 \\
CCACTAT & 0.016 & $0.022,0.014$ & 1.156 & 0.2822 \\
Association-haplotypes suicide & & \\
ACGCCAC & 0.721 & $0.736,0.718$ & 0.429 & 0.5124 \\
CCATTGT & 0.112 & $0.071,0.121$ & 6.261 & $0.0123 *$ \\
CTACTGT & 0.085 & $0.083,0.085$ & 0.019 & 0.8906 \\
ACGCCGT & 0.029 & $0.030,0.029$ & 0.009 & 0.9265 \\
CCACTAT & 0.018 & $0.033,0.014$ & 5.13 & $0.0235 *$ \\
CCACTGT & 0.013 & $0.023,0.011$ & 2.906 & 0.0883 \\
ACATTGT & 0.010 & $0.016,0.009$ & 1.166 & 0.2802 \\
\hline
\end{tabular}

Note: *Indicates association.

Abbreviations: FKBP5, FK506 binding protein 5; BP, bipolar disorder; Freq, frequency.
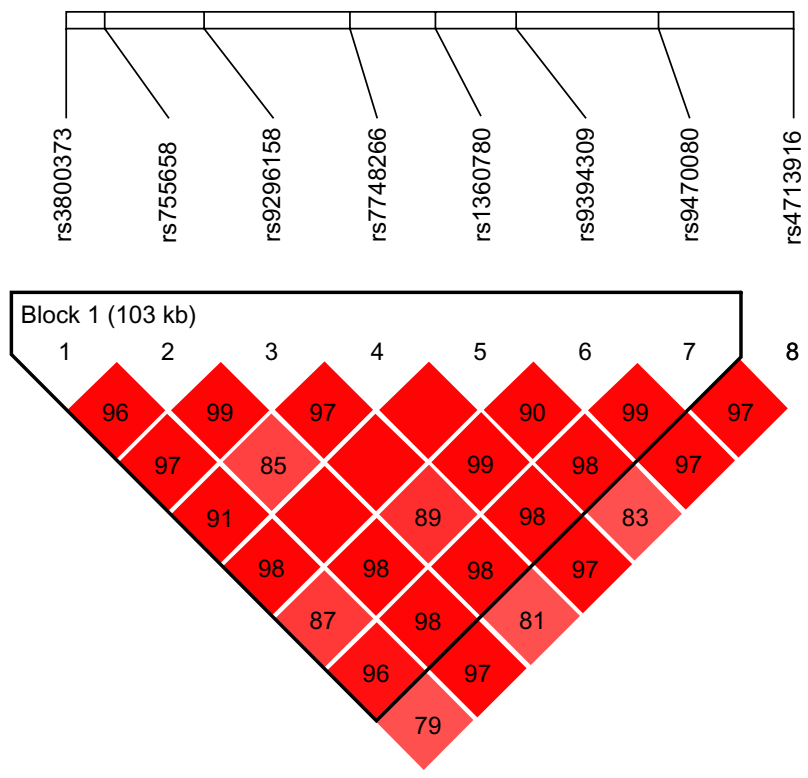

Figure I Relative positions and LD estimates between FKBP5 polymorphisms in the analyzed population-patients with melancholic depression.

Note: Colored squares correspond to D' values with numerical estimates given within the squares.

Associations between variants of the FKBP5 gene and major depression, bipolar disorder, posttraumatic stress disorder, response to antidepressants, and increased recurrence of depression were also found. ${ }^{18-21}$ There have also been studies showing no associations between polymorphisms of the FKBP 5 gene and depression..$^{22}$ In the study performed by our group on the same SNPs of the FKBP5 gene, as in this study, association with major depressive disorder but
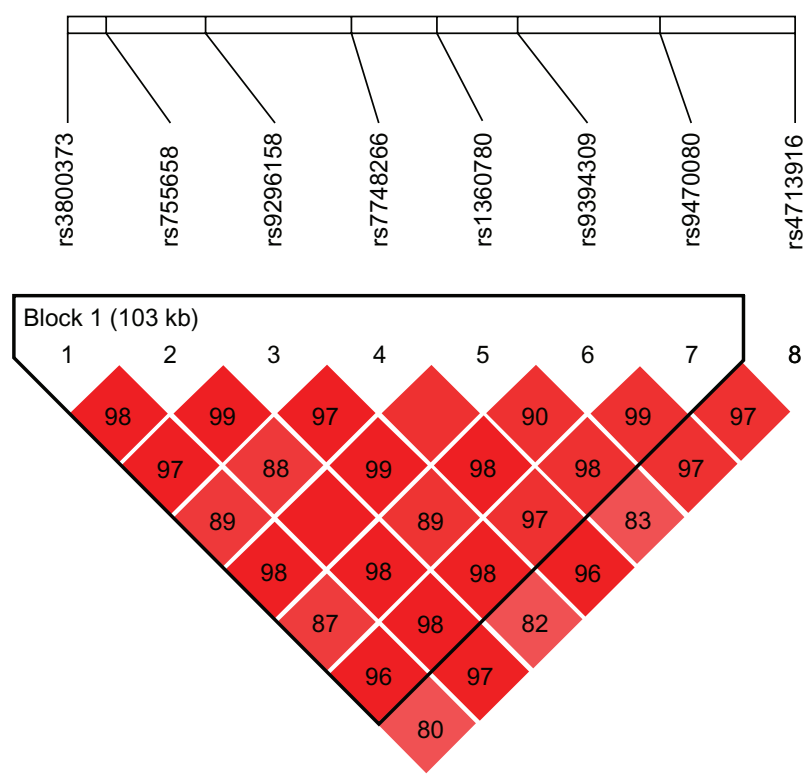

Figure 2 Relative positions and LD estimates between FKBP5 polymorphisms in the analyzed population-patients with psychotic bipolar disorder.

Note: Colored squares correspond to D' values with numerical estimates given within the squares. 


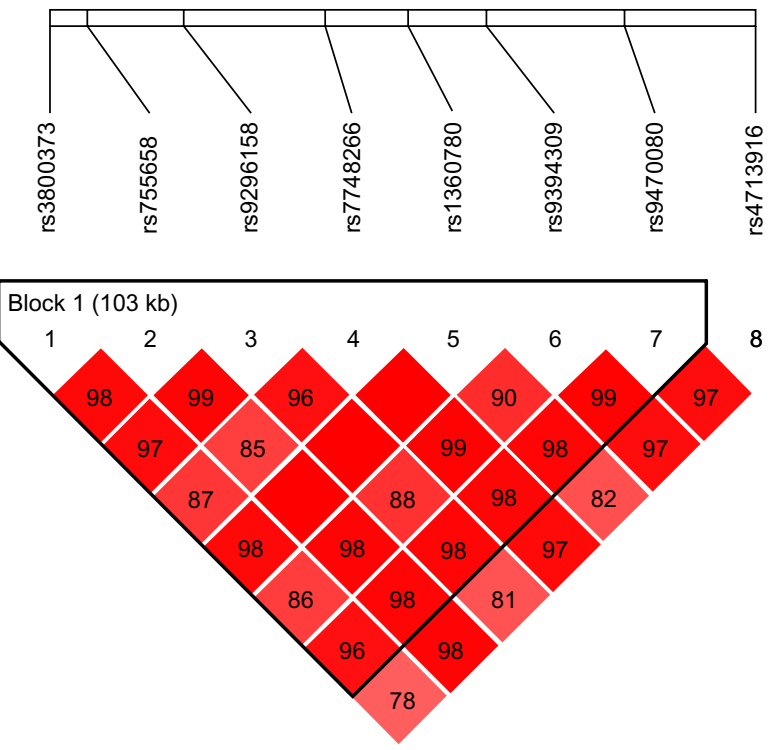

Figure 3 Relative positions and LD estimates between FKBP5 polymorphisms in the analyzed population-bipolar patients with suicide attempts.

Note: Colored squares correspond to D' values with numerical estimates given within the squares.

not with bipolar disorder was found. It was also proved that clinical differences, such as an increase in previous suicide attempts, are occurring in carriers of the risk allele of the FKBP5 gene. $^{4}$ One previous study showed male-specific association of the homozygous genotype of the rs1360780 polymorphism; ${ }^{18}$ however, other studies have not observed a sex-specific effect of FKBP5 in psychiatric disorders. ${ }^{23,24}$

To the best of our knowledge, there have been no studies so far on melancholic depression and psychotic features in the course of bipolar disorder in relation to polymorphisms of the FKBP5 gene. The lack of association found in the group of melancholic patients is in line with our previously published paper showing no association with the other genes (NR3C1, AVPR1B, CRHR1) involved in the HPA axis..$^{25}$ Those results are interesting, because it was the melancholic subtype of depression that was strongly correlated with HPA-axis disturbances.

The results showing no association with the FKBP5 gene and bipolar disorder with psychotic features are also in line with our previous study showing no association with the $N R 3 C 1$ gene coding the GR. In the same study, we also proved association between polymorphisms in the $A V P R 1 B$ and $C R H R 1$ genes and psychotic features in the course of bipolar disorder. In the study performed by Collip et al, which investigated patients with psychotic disorder, their healthy siblings, and controls, an association between trauma and psychosis was apparent. An interaction between the two FKBP5 SNPs rs9296158 and rs4713916 and childhood trauma in models of psychotic symptoms was found. ${ }^{26}$ Supporting this hypothesis
Table 4 Alleles for FKBP5 gene and subtypes of BP disorder

\begin{tabular}{|c|c|c|c|c|c|}
\hline Lp & SNP & $\begin{array}{l}\text { Assoc } \\
\text { allele }\end{array}$ & $\begin{array}{l}\text { Case: control } \\
\text { freq }\end{array}$ & Chi-square & $P$-value \\
\hline \multicolumn{6}{|c|}{ Association-alleles melancholic } \\
\hline I & rs3800373 & C & $0.244,0.240$ & 0.017 & 0.897 \\
\hline 2 & rs755658 & $\mathrm{T}$ & $0.100,0.091$ & 0.201 & 0.6543 \\
\hline 3 & rs9296I58 & $A$ & $0.250,0.247$ & 0.008 & 0.9267 \\
\hline 4 & rs7748266 & C & $0.898,0.865$ & 2.177 & $0.140 \mid$ \\
\hline 5 & rsl360780 & C & $0.754,0.750$ & 0.018 & 0.8921 \\
\hline 6 & rs9394309 & A & $0.760,0.734$ & 0.727 & 0.3939 \\
\hline 7 & rs9470080 & $\mathrm{T}$ & $0.278,0.277$ & 0.001 & 0.9744 \\
\hline 8 & rs4713916 & G & $0.766,0.743$ & 0.603 & 0.4373 \\
\hline \multicolumn{6}{|c|}{ Association-alleles psychotic } \\
\hline I & rs3800373 & C & $0.262,0.240$ & 0.764 & 0.382 \\
\hline 2 & rs755658 & $\mathrm{T}$ & $0.094,0.091$ & 0.036 & 0.8504 \\
\hline 3 & rs9296158 & $A$ & $0.27 I, 0.247$ & 0.896 & 0.3439 \\
\hline 4 & rs7748266 & $\mathrm{T}$ & $0.155,0.135$ & 0.96 & 0.3271 \\
\hline 5 & rs|360780 & $\mathrm{T}$ & $0.276,0.250$ & 1.097 & 0.295 \\
\hline 6 & rs9394309 & G & $0.294,0.266$ & 1.182 & 0.2769 \\
\hline 7 & rs9470080 & $\mathrm{T}$ & $0.319,0.277$ & 2.627 & 0.1051 \\
\hline 8 & rs4713916 & A & $0.282,0.257$ & 1.002 & 0.3168 \\
\hline \multicolumn{6}{|c|}{ Association-alleles suicide } \\
\hline I & rs3800373 & A & $0.790,0.760$ & 1.203 & 0.2728 \\
\hline 2 & rs755658 & C & $0.916,0.909$ & 0.156 & 0.6924 \\
\hline 3 & rs9296158 & G & $0.774,0.753$ & 0.585 & 0.4443 \\
\hline 4 & rs7748266 & C & $0.907,0.865$ & 3.905 & $0.048 I^{*}$ \\
\hline 5 & rsl360780 & C & $0.764,0.750$ & 0.245 & 0.6208 \\
\hline 6 & rs9394309 & A & $0.767,0.734$ & 1.35 & 0.2453 \\
\hline 7 & rs9470080 & C & $0.733,0.723$ & 0.128 & 0.7202 \\
\hline 8 & rs4713916 & G & $0.780,0.743$ & $1.83 \mathrm{I}$ & 0.176 \\
\hline
\end{tabular}

is a study showing that mice lacking the FKBP5 gene were less vulnerable to the adverse effect of social defeat. ${ }^{2}$

A possible reason for discrepancies between the results obtained in this study and other results may be different trauma history in each patient, which could lead to epigenetic differences in the FKBP5 locus and consequently differentiate the genotype effect. ${ }^{27}$ Another reason for the inconsistent results may be the large degree of linkage disequilibrium in FKBP5 and the confined knowledge about the functionality of these SNPs. ${ }^{26,28,29}$

In this context, the main limitation of our study is the absence of data about previous trauma exposure. However, we aimed to examine the narrowly defined phenotype without including environmental interactions. Our study has several other limitations, eg, the fact that $40 \%$ of the control group was psychiatrically screened, whereas the remaining control subjects consisted of blood donors that were screened only for somatic disorders; however, taking into account the prevalence of bipolar disorder in the general population, which is about $2 \%$, the risk of involving bipolar disorder patients in the control group is rather low. Another limitation of our study is the small sample size and the low power of the analyzed 
SNPs, which of course can lead to false-positive results, so further validation and replication of the present findings is still needed.

In conclusion, the results of our study suggest the involvement of haplotypes of the FKBP5 gene in the etiology of suicidal behavior, but not in the etiology of melancholic or psychotic features occurring in the course of bipolar illness.

\section{Acknowledgment}

This study was supported by the Polish Ministry of Science and Higher Education, grant IP 2011053771.

\section{Disclosure}

The authors report no conflicts of interest in this work.

\section{References}

1. Guidotti G, Calabrese F, Anacker C, Racagni G, Pariante CM, Riva MA. Glucocorticoid receptor and FKBP5 expression is altered following exposure to chronic stress: modulation by antidepressant treatment. Neuropsychopharmacology. 2013;38:616-627.

2. Hartmann J, Wagner KV, Liebl C, et al. The involvement of FK506binding protein 51 (FKBP5) in the behavioral and neuroendocrine effects of chronic social defeat stress. Neuropharmacology. 2012;62: 332-339.

3. Pérez-Ortiz JM, García-Gutiérrez MS, Navarrete F, Giner S, Manzanares J. Gene and protein alterations of FKBP5 and glucocorticoid receptor in the amygdala of suicide victims. Psychoneuroendocrinology. 2013;38:1251-1258.

4. Menke A, Klengel T, Rubel J, et al. Genetic variation in FKBP5 associated with the extent of stress hormone dysregulation in major depression. Genes Brain Behav. 2013;12(3):289-96.

5. DeRijk R, de Kloet ER. Corticosteroid receptor genetic polymorphisms and stress responsivity. Endocrine. 2005;28:263-270.

6. Derijk RH. Single nucleotide polymorphisms related to HPA axis reactivity. Neuroimmunomodulation. 2009;16:340-352.

7. Zimmermann P, Brück1 T, Nocon A, et al. Interaction of FKBP5 gene variants and adverse life events in predicting depression onset: results from a 10-year prospective community study. Am J Psychiatry. 2011; 168:1107-1116.

8. Ising M, Depping AM, Siebertz A, et al. Polymorphisms in the FKBP5 gene region modulate recovery from psychosocial stress in healthy controls. Eur J Neurosci. 2008;28:389-398.

9. Binder EB, Bradley RG, Liu W, et al. Association of FKBP5 polymorphisms and childhood abuse with risk of posttraumatic stress disorder symptoms in adults. JAMA. 2008;299:1291-1305.

10. Roy A, Gorodetsky E, Yuan Q, Goldman D, Enoch MA. Interaction of FKBP5, a stress-related gene, with childhood trauma increases the risk for attempting suicide. Neuropsychopharmacology. 2010;35:1674-1683.

11. Supriyanto I, Sasada T, Fukutake M, et al. Association of FKBP5 gene haplotypes with completed suicide in the Japanese population. Prog Neuropsychopharmacol Biol Psychiatry. 2010;35:252-256.

Neuropsychiatric Disease and Treatment

\section{Publish your work in this journal}

Neuropsychiatric Disease and Treatment is an international, peerreviewed journal of clinical therapeutics and pharmacology focusing on concise rapid reporting of clinical or pre-clinical studies on a range of neuropsychiatric and neurological disorders. This journal is indexed on PubMed Central, the 'PsycINFO' database and CAS.
12. Cavalli-Sforza L. The History and Geography of Human Genes. Princeton (NJ): Princeton University Press; 1994.

13. First MB, Spitzer RL, Gibbon M, Williams JB. Structured Clinical Interview for DSM-IV Axis I Disorders. New York: New York State Psychiatric Institute; 1995.

14. Miller SA, Dykes DD, Polesky HF. A simple salting out procedure for extracting DNA from human nucleated cells. Nucleic Acids Res. 1988;16:1215.

15. Barrett JC, Fry B, Maller J, Daly MJ. Haploview: analysis and visualization of LD and haplotype maps. Bioinformatics. 2005;21:263-265.

16. Fardo DW, Becker KD, Bertram L, Tanzi RE, Lange C. Recovering unused information in genome-wide association studies: the benefit of analyzing SNPs out of Hardy-Weinberg equilibrium. Eur J Hum Genet. 2009;17:1676-1682.

17. Brent D, Melhem N, Ferrell R, et al. Association of FKBP5 polymorphisms with suicidal events in the Treatment of Resistant Depression in Adolescents (TORDIA) study. Am J Psychiatry. 2010;167:190-197.

18. Lavebratt C, Aberg E, Sjoholm LK, Forsell Y. Variations in FKBP5 and BDNF genes are suggestively associated with depression in a Swedish population-based cohort. J Affect Disord. 2010;125:249-255.

19. Willour VL, Chen H, Toolan J, et al. Family-based association of FKBP5 in bipolar disorder. Mol Psychiatry. 2008;14:261-268.

20. Mehta D, Gonik M, Klengel T, et al. Using polymorphisms in FKBP5 to define biologically distinct subtypes of posttraumatic stress disorder: evidence from endocrine and gene expression studies. Arch Gen Psychiatry. 2011;68:901-910.

21. Binder EB, Salyakina D, Lichtner P, et al. Polymorphisms in FKBP5 are associated with increased recurrence of depressive episodes and rapid response to antidepressant treatment. Nat Genet. 2004;36:1319-1325.

22. Papiol S, Arias B, Gastó C, Gutiérrez B, Catalán R, Fañanás L. Genetic variability at HPA axis in major depression and clinical response to antidepressant treatment. J Affect Disord. 2007;104:83-90.

23. Velders FP, Kuningas M, Kumari M, et al. Genetics of cortisol secretion and depressive symptoms: a candidate gene and genome wide association approach. Psychoneuroendocrinology. 2011;36:1053-1061.

24. Mahon PB, Zandi PP, Potash JB, Nestadt G, Wand GS. Genetic association of FKBP5 and CRHR1 with cortisol response to acute psychosocial stress in healthy adults. Psychopharmacology (Berl). 2013;227:231-241.

25. Leszczyńska-Rodziewicz A, Szczepankiewicz A, Dmitrzak-Węglarz M, Skibińska M, Hauser J. Association between functional polymorphism of the AVPR1b gene and polymorphism rs1293651 of the CRHR1 gene and bipolar disorder with psychotic features. JAffect Disord. 2012;138: 490-493.

26. Collip D, Myin-Germeys I, Wichers M, et al. FKBP5 as a possible moderator of the psychosis-inducing effects of childhood trauma. $\mathrm{Br} J$ Psychiatry. 2013;202:261-268.

27. Klengel T, Mehta D, Anacker C. Allele-specific FKBP5 DNA demethylation mediates gene-childhood trauma interactions. Nat Neurosci. 2013; 16:33-41.

28. Binder EB, Künzel, HE, Nickel T, et al. HPA-axis regulation at inpatient admission is associated with antidepressant therapy outcome in male but not in female depressed patients. Psychoneuroendocrinology. 2009;34:99-109.

29. Binder EB. The role of FKBP5, a co-chaperone of the glucocorticoid receptor in the pathogenesis and therapy of affective and anxiety disorders. Psychoneuroendocrinology. 2009;34 Suppl 1:S186-S195.

\section{Dovepress}

The manuscript management system is completely online and includes a very quick and fair peer-review system, which is all easy to use. Visit http://www.dovepress.com/testimonials.php to read real quotes from published authors. 